\title{
Barbituric Derivative Nanoaggregates with Aggregation-Induced Emission and Mechanofluorochromism
}

\author{
Xi Su, ${ }^{1}$ Hanjun Zhang, ${ }^{1}$ Kaiyue You, ${ }^{1}$ Hua Wang, ${ }^{2}$ Yuezhi Cui $\mathbb{D},{ }^{1}$ and William W. Yu $\mathbb{D}^{2}$ \\ ${ }^{1}$ School of Chemical and Pharmaceutical Engineering, Qilu University of Technology (Shandong Academy of Sciences), \\ Jinan 250353, China \\ ${ }^{2}$ Louisiana State University, Shreveport, Louisiana 71115, USA
}

Correspondence should be addressed to Yuezhi Cui; yuezhicui@163.com and William W. Yu; wyu6000@gmail.com

Received 28 February 2019; Revised 14 May 2019; Accepted 18 August 2019; Published 11 November 2019

Academic Editor: Domenico Acierno

Copyright @ 2019 Xi Su et al. This is an open access article distributed under the Creative Commons Attribution License, which permits unrestricted use, distribution, and reproduction in any medium, provided the original work is properly cited.

Three new nonplanar barbituric derivatives, named as TTB, TTTB, and TOB, were synthesized. The D- $\pi$-A type conjugated compounds showed obvious intramolecular charge transfer (ICT) property, which was evidenced by theoretical calculations and spectral analyses. All of them exhibited aggregation induced emission (AIE) when formed nanoaggregates. These nanoaggregates also showed reversible mechanofluorochromism (MFC). Their red light emission became deep red after grinding and then recovered with dichloromethane fuming. Hence, a strategy to fabricate mechanofluorochromic nanoaggregate phosphors via nonplanar $\pi$-skeleton and steric effect was demonstrated, and these nanophosphors possess potentials for mechanosensors and anticounterfeiting technology.

\section{Introduction}

Conventional organic luminescent materials have little fluorescence due to aggregation (aggregation-caused quenching (ACQ) effect), which severely limits the applications in solid state $[1,2]$. Fortunately, Hong and coworkers discovered a series of compounds and opened a new door for aggregation-induced emission (AIE) [3, 4]. Many research groups have developed strong interest in further designing and synthesizing some AIE organic luminescent materials and have discovered and summarized the interesting properties of AIE materials [5-12]. Though many AIE materials have been reported, however, the relationship between molecule structure and the AIE effect still remains ambiguous. Therefore, it is of great significance to design and synthesize more AIE compounds and explore the structure-property relationship.

In our former work, a series of thiophene derivatives with one, two, and four branches attaching on thiophene, respectively, were synthesized [13]. In these compounds, intramo- lecular charge transfer (ICT) occurred from each branch to thiophene, producing multidirectional polarization body. The relationship between the multidirectional polarization and the AIE effect was studied. It was found that simple one-branched compound with dipolar ICT showed advantage in AIE effect over the multibranched one.

Based on the former work, we further design and synthesize several dipolar compounds constituted by thiophene and barbitural acid in the current submission. In this work, the monomer, dimer, and trimer of thiophenyl serve as electron donor. The emphasis of this work is put on the relationship between the degree of the oligomerization in the electron donor and the AIE effect. The research results show that the compound with the trimer of thiophenyl exhibits the strongest AIE effect. Additionally, these barbituric derivative nanoaggregates exhibited strong mechanofluorochromism (MFC) effects. The detailed results are reported below.

In this paper, three barbituric derivatives were synthesized and exhibited very good AIE property. Piezochromic 


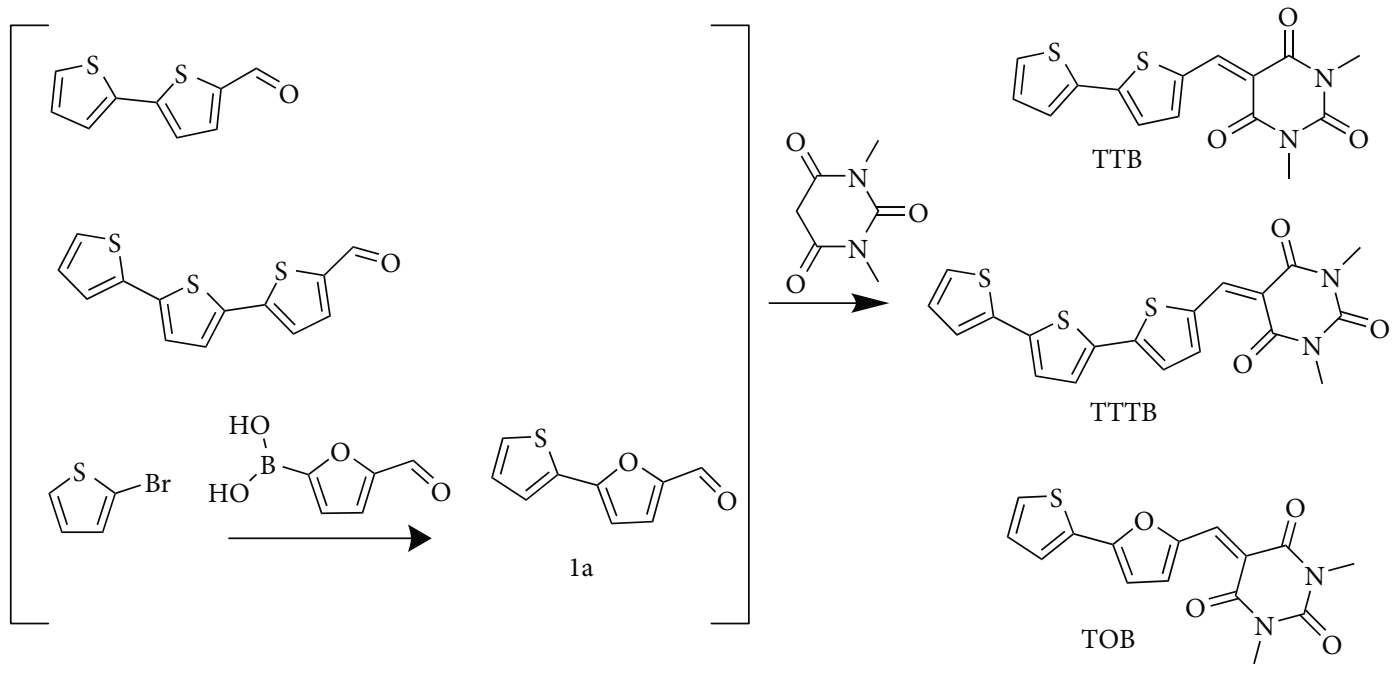

Scheme 1: Synthetic routes towards TTB, TTTB, and TOB compounds.

luminescent materials are a type of force-stimulated responsive materials that change the luminescence color and intensity by varying the physical packing of the molecules [14-17].

\section{Experimental Section}

2.1. Materials and Characterizations. All the reagents were obtained commercially and used without further purification: 1,3-dimethylbarbituric acid (Aladdin, 98\%), 2,2'-bithiophene-5-carboxaldehyde (Macklin, 98\%), and 2,2':5',2"-terthiophene-5-carboxaldehyde (Macklin, 98\%). All the solvents used in this study were purified according to standard methods prior to use. The ${ }^{1} \mathrm{H}$ NMR spectrum was recorded by AVANCZ II 400 spectrometer using chloroform $\left(\mathrm{CDCl}_{3}\right)$ as solvent. The absorption spectrum was recorded using UV-2500 spectrometer. Measuring fluorescence (FL) spectra was recorded by a F-4600 fluorescence spectrophotometer. The fluorescence quantum yields of pure solution and aggregate state were determined relative to Coumarin 307 in ethanol solution as a quantum yield standard $\left(\Phi_{F}\right.$ (fluorescence quantum yield $\left.)=56 \%\right) \quad[18]$. The absorption and emission wavelengths of Coumarin 307 are $395 \mathrm{~nm}$ and $500 \mathrm{~nm}$ in EtOH; since Coumarin 307 has a similar maximum absorption and emission wavelength as the synthesized compound, it was selected as a reference standard to determine the fluorescence quantum yield of TTB, TTTB, and TOB. Theoretical calculations at the B3LYP/6$31 \mathrm{G}$ (d) basis were used to determine the HOMO and LUMO energies for the three compounds.

2.2. Syntheses. The compound of 1a was synthesized according to literature methods [19]. The target luminogens (TTB, TTTB, TOB) were synthesized by the Knoevenagel condensation of aldehyde-based intermediates with 1,3-dimethyl barbituric acid (Scheme 1).

2.2.1. Synthesis of 2,2'Bithiophene-1,3-Dimethylbarbituric Acid (TTB). A mixture of 1,3-dimethylbarbituric acid (0.960 g, $6.18 \mathrm{mmol})$ and 2,2' -bithiophene-5-carboxaldehyde $(1.00 \mathrm{~g}, 5.15 \mathrm{mmol})$ in acetic $(5 \mathrm{~mL})$ and acetic anhydride
$(5 \mathrm{~mL})$ was refluxed for $2 \mathrm{~h}$. The reaction was cooled to room temperature and solid particles were filtered. The product was then purified by recrystallization with acetic acid to give $0.89 \mathrm{~g}$ (90\% yield): ${ }^{1} \mathrm{H} \mathrm{NMR}\left(400 \mathrm{MHz}, \mathrm{CDCl}_{3}\right): \delta 8.65$ $(\mathrm{s}, 1 \mathrm{H}), 7.80(\mathrm{~s}, 1 \mathrm{H}), 7.55(\mathrm{~s}, 1 \mathrm{H}), 7.44(\mathrm{~d}, J=5.4 \mathrm{~Hz}$, $1 \mathrm{H}), 7.37(\mathrm{~d}, J=4.6 \mathrm{~Hz}, 1 \mathrm{H}), 7.12(\mathrm{~s}, 1 \mathrm{H}), 3.43(\mathrm{~s}, 6 \mathrm{H})$ (Figure S1); ${ }^{13} \mathrm{C}$ NMR $\left(151 \mathrm{MHz}, \mathrm{CDCl}_{3}\right): \delta 162.18,157.65$, $134.69,130.97,130.33,127.52,125.40,124.62,124.45$, 124.30, 123.78, 28.62, 27.97 (Figure S4).

2.2.2. Synthesis of $2,2^{\prime}: 5^{\prime}, 2^{\prime \prime}$-Terthiophene-1,3Dimethylbarbituric Acid (TTTB). A mixture of 1,3-dimethylbarbituric acid $(0.670 \mathrm{~g}, 4.35 \mathrm{mmol})$ and $2,2^{\prime}: 5^{\prime}, 2^{\prime \prime}$-terthiophene-5-carboxaldehyde $(1.00 \mathrm{~g}, 3.62 \mathrm{mmol})$ in ethanol $(10 \mathrm{~mL})$ and two drops of acetic acid was refluxed for $4 \mathrm{~h}$. The reaction was cooled to room temperature, and the solid particles were filtered. The product was then purified by recrystallization with acetic acid to give $0.85 \mathrm{~g}$ ( $83 \%$ yield): ${ }^{1} \mathrm{H}$ NMR $\left(400 \mathrm{MHz}, \mathrm{CDCl}_{3}\right): \delta 8.63(\mathrm{~d}, J=6.6 \mathrm{~Hz}, 1 \mathrm{H})$, $7.80(\mathrm{~d}, J=4.6 \mathrm{~Hz}, 1 \mathrm{H}), 7.47(\mathrm{~d}, J=4.2 \mathrm{~Hz}, 1 \mathrm{H})$, $7.36(\mathrm{~d}, \quad J=4.2 \mathrm{~Hz}, 1 \mathrm{H}), 7.24(\mathrm{~s}, 2 \mathrm{H}), 7.19$ (s, 1H), 7.06 $(\mathrm{d}, J=5.3 \mathrm{~Hz}, 1 \mathrm{H}), 3.43(\mathrm{~s}, 6 \mathrm{H})$ (Figure $\mathrm{S} 2) ;{ }^{13} \mathrm{C} \mathrm{NMR}$ $\left(101 \mathrm{MHz}, \mathrm{CDCl}_{3}\right): \delta 162.22,161.56,153.11,150.90,147.99$, $146.47,135.79,134.99,128.14,127.59,126.37,124.14$, 108.50, 28.39, 27.63 (Figure S5).

2.2.3. Synthesis of 5-Thiophen-2-yl-Furan-2-Carbaldehyde1,3-Dimethylbarbituric Acid (TOB). A mixture of 1,3dimethylbarbituric acid $(1.05 \mathrm{~g}, 6.74 \mathrm{mmol})$ and $1 \mathrm{a}(1.00 \mathrm{~g}$, $5.62 \mathrm{mmol})$ in ethanol $(10 \mathrm{~mL})$ and two drops of acetic acid was refluxed for $6 \mathrm{~h}$. The reaction was cooled to room temperature and the solid particles were filtered. The product was then purified by recrystallization with acetic acid to give $0.58 \mathrm{~g}$ (61\% yield): ${ }^{1} \mathrm{H} \mathrm{NMR}\left(600 \mathrm{MHz}, \mathrm{CDCl}_{3}\right): \delta 8.76$ $(\mathrm{d}, J=1.1 \mathrm{~Hz}, 1 \mathrm{H}), 8.51(\mathrm{~s}, 1 \mathrm{H}), 8.01(\mathrm{dt}, J=5.1,1.3 \mathrm{~Hz}$, $1 \mathrm{H}), 7.91(\mathrm{dd}, J=3.9,1.3 \mathrm{~Hz}, 1 \mathrm{H}), 7.29(\mathrm{dd}, J=5.1,3.8 \mathrm{~Hz}$, $1 \mathrm{H}), 7.26(\mathrm{~s}, 1 \mathrm{H}), 3.43\left(\mathrm{~d}, J=3.8 \mathrm{~Hz}, 6 \mathrm{H}\right.$ ) (Figure S3); ${ }^{13} \mathrm{C}$ NMR $\left(151 \mathrm{MHz}, \mathrm{CDCl}_{3}\right): \delta 162.69,161.77,151.35,149.07$, 145.46, 141.91, 128.25, 110.56, 28.95, 28.16 (Figure S6). 


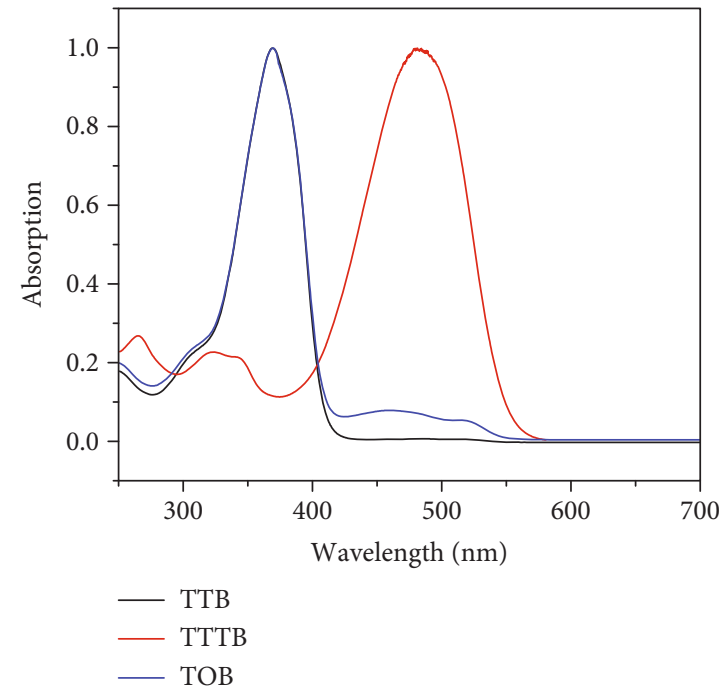

(a)

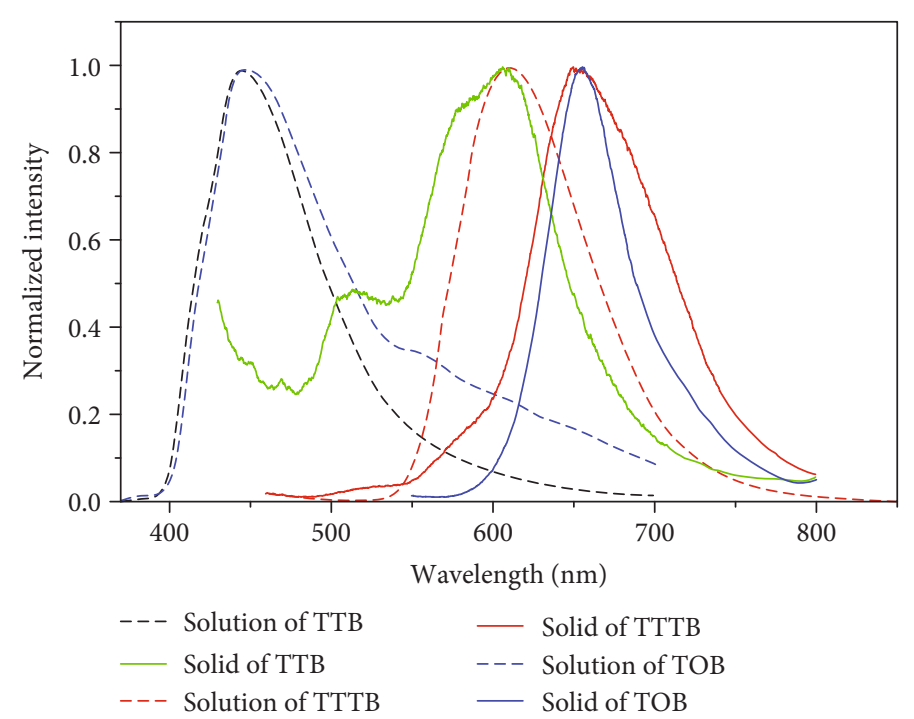

(b)

FIGURE 1: (a) Absorption and (b) emission spectra of three compounds in THF solution (concentration: $1.0 \times 10^{-5} \mathrm{~mol} / \mathrm{L}$; excitation wavelength, TTB: $360 \mathrm{~nm}$, TTTB: $480 \mathrm{~nm}$, and TOB: $360 \mathrm{~nm}$ ) and solid state (excitation wavelength, TTB: $410 \mathrm{~nm}$, TTTB: $530 \mathrm{~nm}$, and TOB: $440 \mathrm{~nm})$.

\section{Results and Discussion}

3.1. Optical Properties. As shown in Figure 1, the absorption peak wavelength $\left(\lambda_{\mathrm{a}}\right)$ and emission wavelength $\left(\lambda_{\mathrm{em}}\right)$ of TTB and TOB are similar in THF solution, indicating that both of the molecules have similar distribution of electronic levels. The $\lambda_{\mathrm{a}}$ and $\lambda_{\mathrm{em}}$ for TTTB are much longer than those of TTB and TOB, which may be due to that TTTB is a larger conjugated molecule with an additional thienyl in the electron donor (Figures 1(a) and 1(b)). As shown in Figure 1(b), the emission wavelengths of the three compounds are 585, 654, and $640 \mathrm{~nm}$, respectively. Obviously, in the solid state, the emission wavelength of TTB shows a blue shift compared to TTTB and TOB. These results have been proved by theoretically calculated.

3.2. AIE Activities. The photoluminescence (PL) behaviors of compounds TTB, TTTB, and TOB were investigated in $\mathrm{THF} / \mathrm{H}_{2} \mathrm{O}$ mixed solvent (Figure 2) with the concentration of all the three compounds of $1.0 \times 10^{-5} \mathrm{~mol} / \mathrm{L}$. From Figure 2(a), it can be clearly seen that the three compounds have obvious AIE properties which show weak fluorescence in pure THF solution and strong emission in the aggregate state. For Figure 2(b), when $f_{w} \leq 80 \%$ for TTB and TOB, as well as $f_{\mathrm{w}} \leq 70 \%$ for TTTB, it is obvious that the fluorescence intensity increases as $f_{\mathrm{w}}$ is increasing, accompanied by a red shift of the emission wavelength $\left(\lambda_{\mathrm{em}}\right)$ which may be caused by intramolecular charge transfer (ICT). As the water content increases, the fluorescence intensity first increases and then decreases, and the emission wavelength is always red-shifted. To verify the ICT mechanism, emission spectra were recorded in different solvents with various polarities. As can be seen from Figure 3, the emission wavelengths of the barbituric acid derivatives TTB, TTTB, and TOB were red-shifted with the increase of the solvent polarity. This phenomenon is typical behavior of molecules with ICT nature. The theoretical calculation results are consistent with this phenomenon (Figure 4). When $f_{\mathrm{w}}$ increases, the molecules begin to accumulate in a limited space and, to some extent, limit the free rotation of the intramolecular rotatable groups, which makes the radiation transition pathway active and leads to enhanced fluorescence emission [20-22]. However, when $f_{\mathrm{w}}>80 \%$ for TTB and TOB, as well as $f_{\mathrm{w}}>70 \%$ for TTTB, molecules began to precipitate, resulting in weak emission and blue shifts of the $\lambda_{\mathrm{em}}[23,24]$. Similarly, it can be observed from the photographs of TTB, TTTB, and TOB (Figure 5), with the $f_{\mathrm{w}}$ ranging from 0 to $90 \%$, all the three compounds are AIE active.

The AIE index $I_{\mathrm{m}} / I_{0}$, where $I_{\mathrm{m}}$ is the maximum fluorescence intensity of the nanoaggregate solution $\left(0<f_{\mathrm{w}}<100 \%\right)$, and $I_{0}$ is the fluorescence intensity when $f_{\mathrm{w}}=0 \%$, is generally used to quantify the AIE activity [25]. From Figure 2(b), obviously, TTTB exhibits enhanced AIE effect compared to TTB and TOB: in aggregation solution, the luminescence intensity of TTTB increased by 20 -fold $\left(f_{\mathrm{w}}=70 \% / f_{\mathrm{w}}=0 \%\right)$, with the fluorescence quantum yield $\left(\Phi_{F}\right)$ increasing from $1.5 \%$ to $14.9 \%$. However, the maximum luminescence enhancement of TTB $\left(f_{\mathrm{w}}=80 \% / f_{\mathrm{w}}=0 \%\right)$ is only 4 -fold ( $\Phi_{F}: 0.3 \%$ to $1.6 \%$ ) and the maximum emission enhancement of TOB $\left(f_{\mathrm{w}}=80 \% / f_{\mathrm{w}}=0 \%\right)$ is only 6 -fold ( $\Phi_{F}: 0.4 \%$ to $2.1 \%$ ). TTTB shows the highest AIE index. This may be attributed to two facts: (i) the energy gap between HOMO and LUMO of TTTB is the smallest of the three compounds, resulting in the strongest emission; (ii) oligothiophene is a stronger electron donor than the other two compounds, which may lead to a stronger ICT in TTTB, 

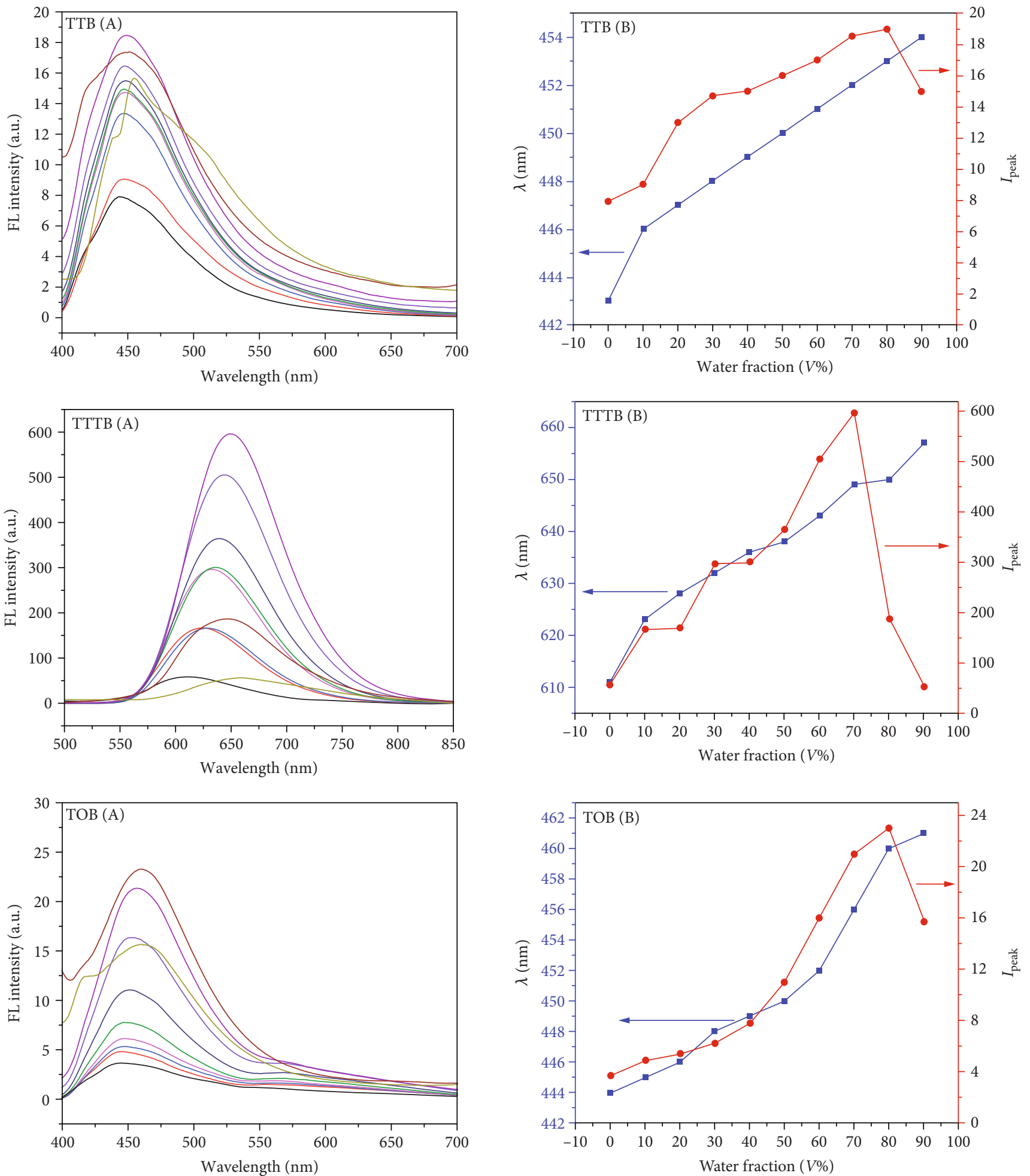

$\begin{array}{rlrl}-f_{\mathrm{w}} & =0 \% & -f_{\mathrm{w}} & =20 \% \\ -f_{\mathrm{w}} & =10 \% & -f_{\mathrm{w}} & =30 \% \\ -f_{\mathrm{w}} & =80 \% & -f_{\mathrm{w}} & =90 \% \\ -f_{\mathrm{w}} & =40 \% & -f_{\mathrm{w}}=60 \% \\ -f_{\mathrm{w}}=50 \% & -70 \%\end{array}$

Figure 2: (a) FL spectra of TTB, TTTB, and TOB in THF/ $\mathrm{H}_{2} \mathrm{O}$ with different water fractions $\left(f_{\mathrm{w}}\right)$. (b) FL peak location (blue) and emission intensity (red) of TTB, TTTB, and TOB versus $f_{\mathrm{w}}$ in THF/ $\mathrm{H}_{2} \mathrm{O}$ (concentration: $1.0 \times 10^{-5} \mathrm{~mol} / \mathrm{L}$; excitation wavelength, TTB: $360 \mathrm{~nm}$, TTTB: $480 \mathrm{~nm}$, and TOB: $360 \mathrm{~nm}$ ). 

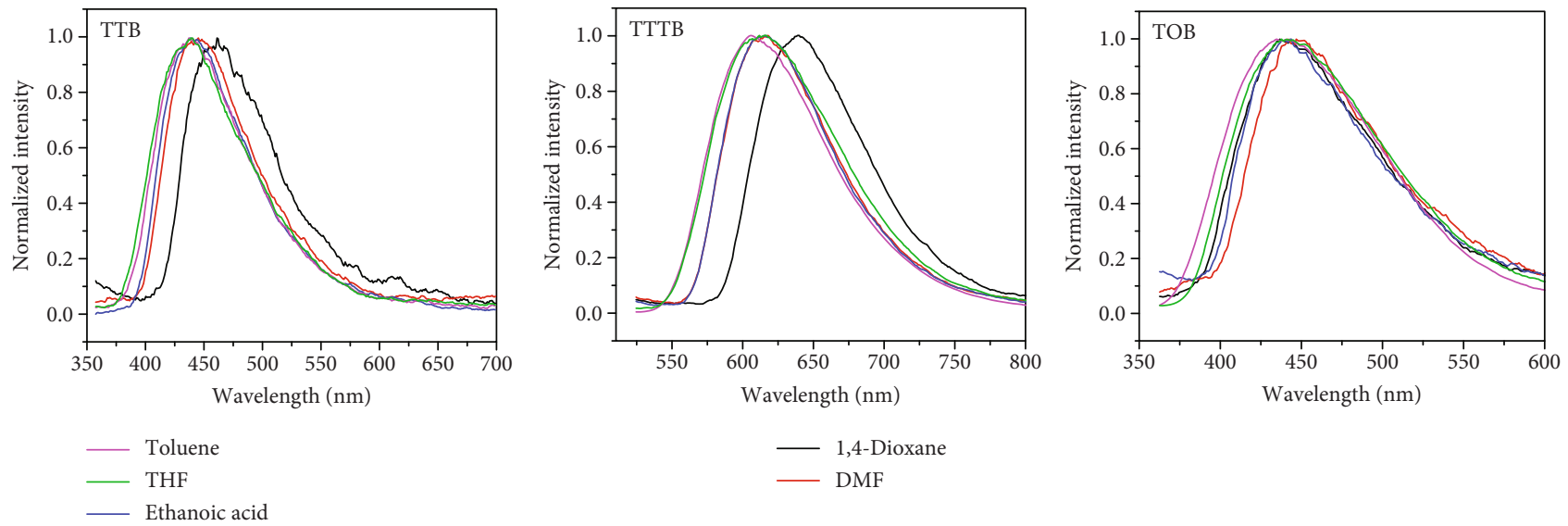

FIgURE 3: PL spectra of TTB, TTTB, and TOB in different solvents with varying polarities (concentration: $1.0 \times 10^{-5} \mathrm{~mol} / \mathrm{L}$; excitation wavelength, TTB: $360 \mathrm{~nm}$, TTTB: $480 \mathrm{~nm}$, and TOB: $360 \mathrm{~nm}$ ).

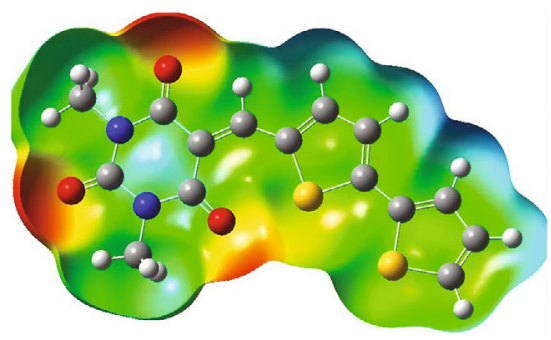

(a)

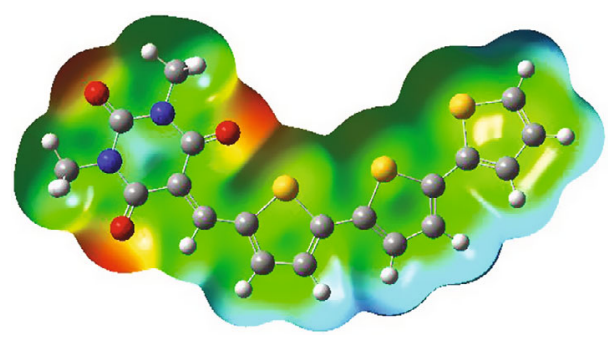

(b)

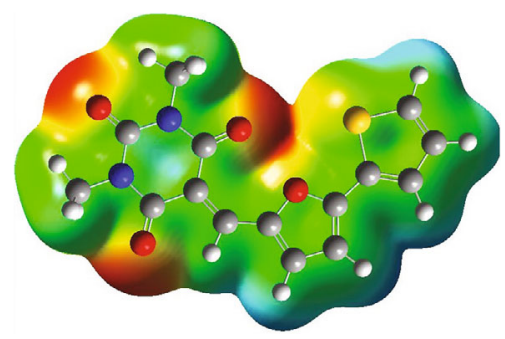

(c)

FIGURE 4: Plots of the electrostatic potential for TTB (a), TTTB (b), and TOB (c) with B3LYP/6-31G (d) on the isodensity surface of 0.001. The color is coded as red for strong negative and blue for strong positive.
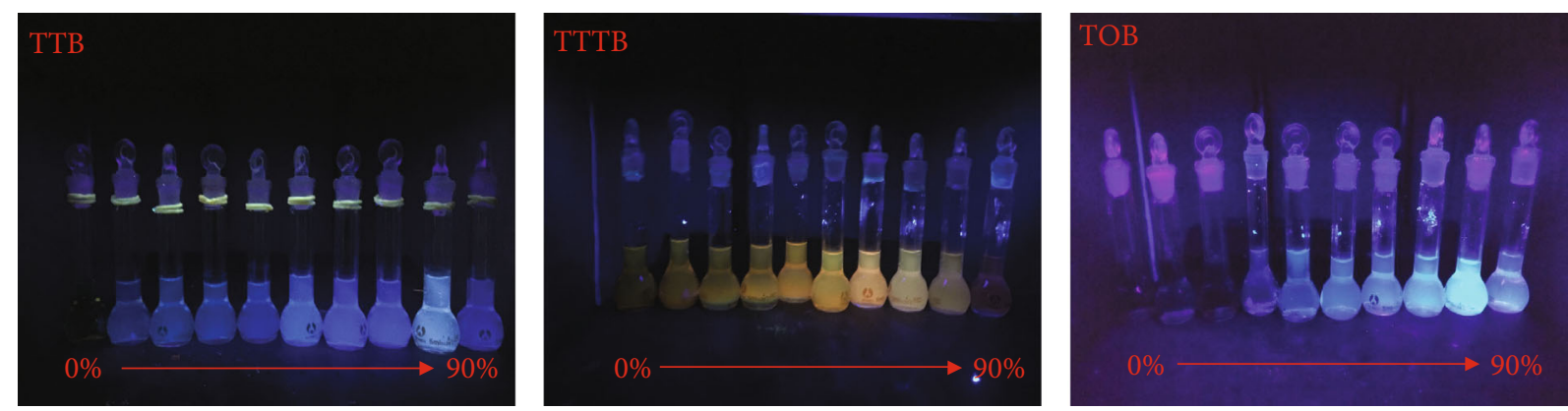

Figure 5: Photographs of TTB, TTTB, and TOB at $f_{\mathrm{w}}=0-90 \%$ under $365 \mathrm{~nm}$ UV light illumination.

meaning that enhanced electrostatic interaction between TTTB molecules that limits the intramolecular rotation of some bonds in the nanoaggregate state [26-28].

The morphology and size of the nanoaggregates in the $\mathrm{THF} / \mathrm{H}_{2} \mathrm{O}$ with different $f_{\mathrm{w}}$ were observed by scanning electron microscopy (SEM) and dynamic light scattering (DLS) (Figure 6), which show the formation of nanoaggregates with relatively narrow sized distributions. As shown in Figure 6, the average diameters $(d)$ of the nanoaggregates for TTB, TTTB, and TOB with their strongest emission intensities (when $f_{\mathrm{w}}=80 \%, 70 \%$, and $80 \%$ ) are 140, 200, $130 \mathrm{~nm}$, respectively.
3.3. Theoretical Calculations. As shown in Figure 7, the HOMO-LUMO gaps for TTB, TTTB, and TOB are 3.07, 2.71, and $3.07 \mathrm{eV}$, respectively. The $\mathrm{HOMO}$ mainly localizes in the donor furan or thiophene units, and the LUMO distributes over all the molecules; it was clearly confirmed that a charge transfer effect occurred in the molecules [29]. As shown in Figure 7, the visualized electrostatic potential exhibited intramolecular charge transfer with a positive region (blue) on the donor moiety and a negative region (red) on the acceptor moiety. This theoretical result illustrates the transfer of electrons from the donor to the acceptor moiety, corresponding to the observed optical properties. 

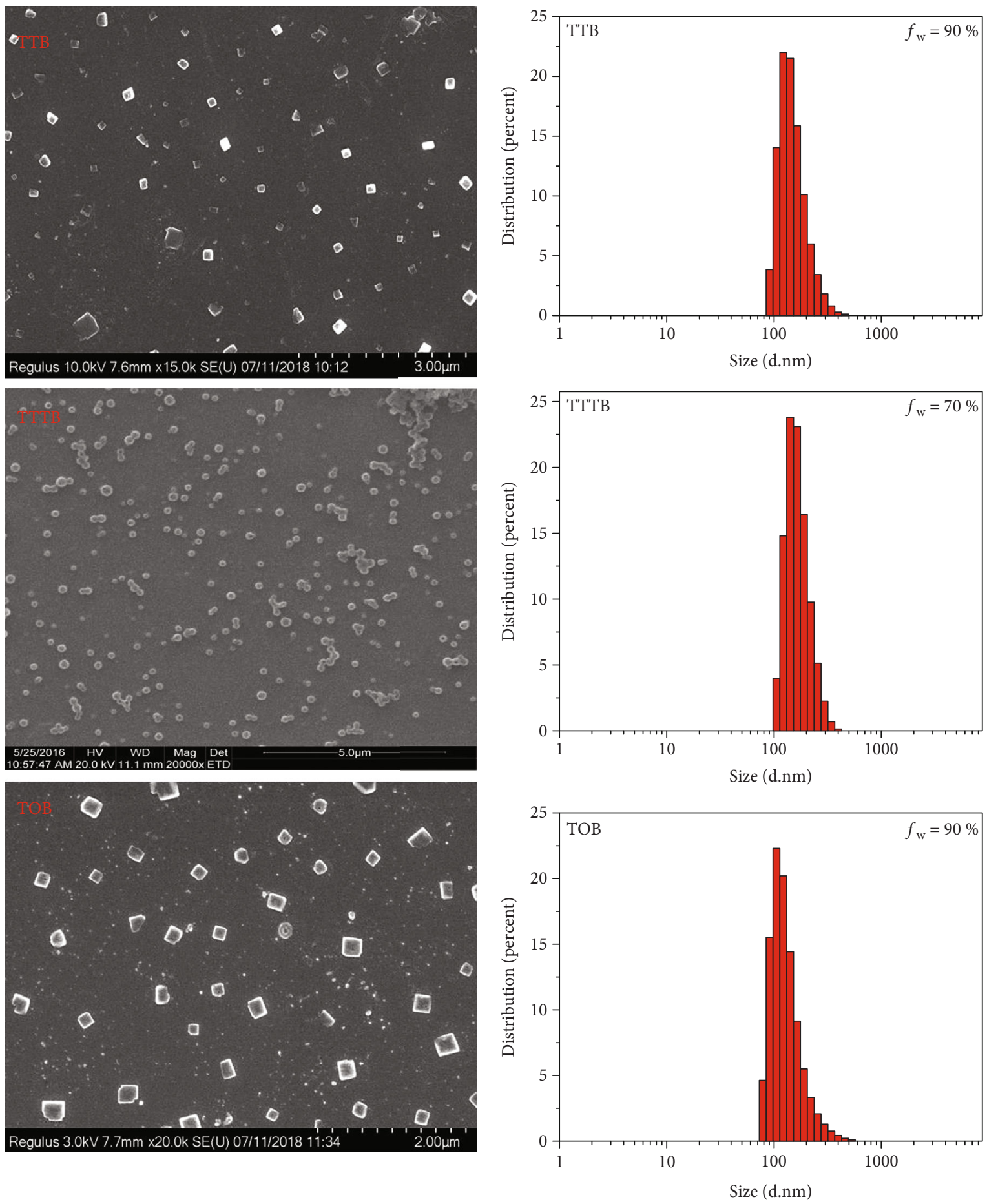

(a)

(b)

Figure 6: SEM images (a) and DLS sizes (b) of TTB, TTTB, and TOB in THF/ $\mathrm{H}_{2} \mathrm{O}$ (nanoaggregate state) at a concentration of $1 \times 10^{-5} \mathrm{mM}$.

3.4. Mechanofluorochromic Properties. Based on the MFC characteristics of nonplanar AIE molecules, the MFC performances of TTB, TTTB, and TOB with helical structure were investigated. As shown in Figures 8 and 9, TTB, TTTB, and TOB are red powder (nanoaggregates) with red fluorescence $\left(\lambda_{\max }=585,654\right.$, and 640 , respectively) under $365 \mathrm{~nm} \mathrm{UV}$ excitation. After grinding, the color of TTB and TOB only changed a little ( $\lambda_{\max }=593$ and 645, respectively), indicating that the MFC activities for both TTB and TOB are very weak and can be negligible. However, the color of TTTB became deep red with a red shift of $26 \mathrm{~nm}$. The color change of TTTB before and after grinding can be easily observed with naked eyes. 


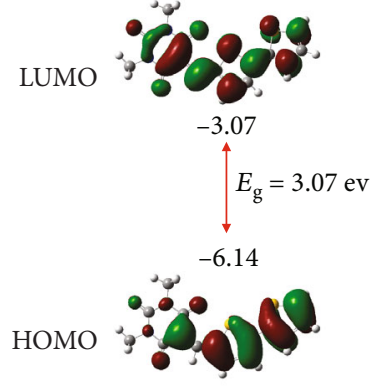

TTB

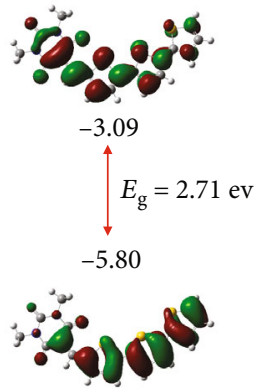

TTTB

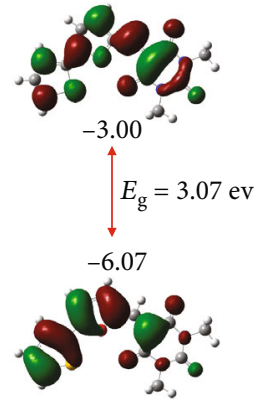

TOB

FIGURE 7: Energy levels of HOMO and LUMO, energy gap, and electron cloud distribution of three molecules calculated by B3LYP/6-31G (d) program.
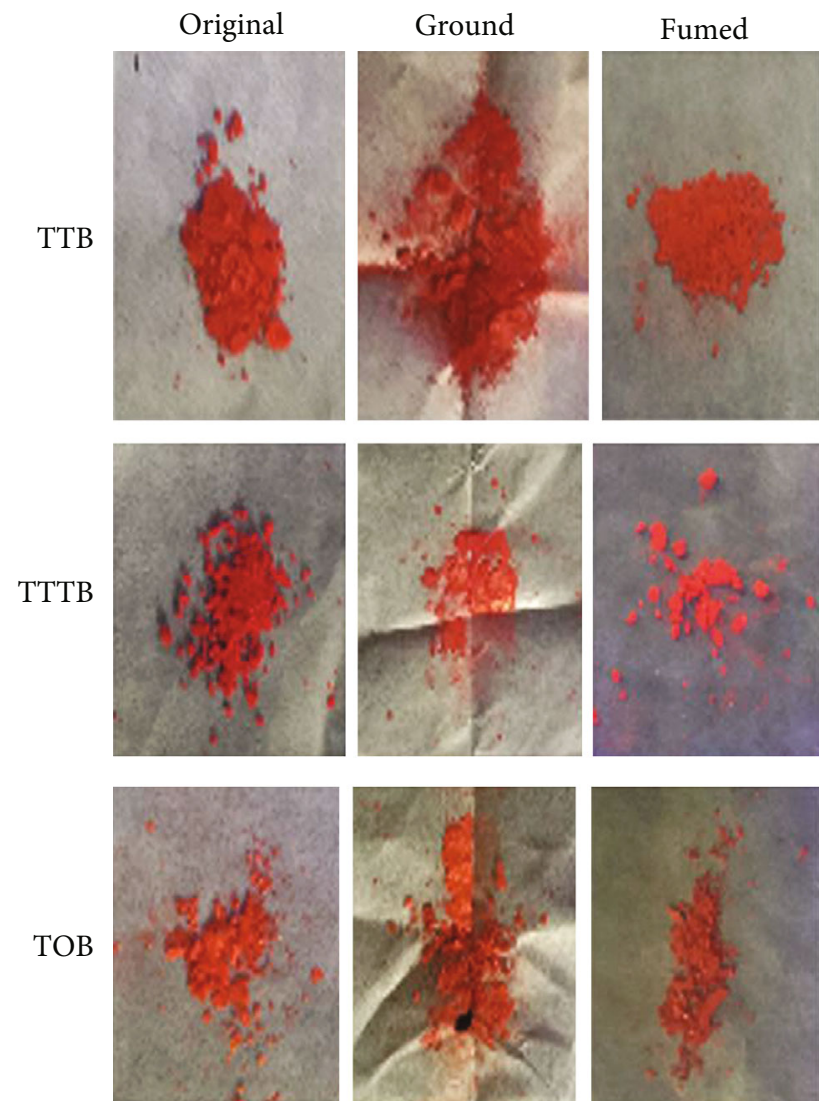

Figure 8: Photos of TTB, TTTB, and TOB and color changes under grinding and fuming stimuli.

After grinding, the three ground powders were exposed to $\mathrm{CH}_{2} \mathrm{Cl}_{2}$ vapor at room temperature. As can be seen from Figure 8, the fluorescent color of the compound after the fume almost recovered to its original state. In particular, the color change of TTTB is more obvious, its fluorescent color changes from a ground deep-red to orange-red after fuming, and the process is completely reversible.

Powder X-ray diffraction (XRD) were used to further explore the MFC mechanism of these nanoaggregates. As shown in Figure 10, all of the original nanoaggregate samples had sharp and strong peaks, but the intensity of the peaks decreased or even disappeared after grinding. The result implies that the molecular morphology changed from crystalline to amorphous [30-32], and red shift of $\lambda_{\mathrm{em}}$ was attributed to the planarization induced by grinding. AIE molecules usually adopt a twisted configuration due to the steric hindrance between moieties and the formation of intermolecular cavities with a loose packing mode. The binding energy released after grinding and accompanied by the destruction of crystal lattice. After fumed with methylene chloride gas, 

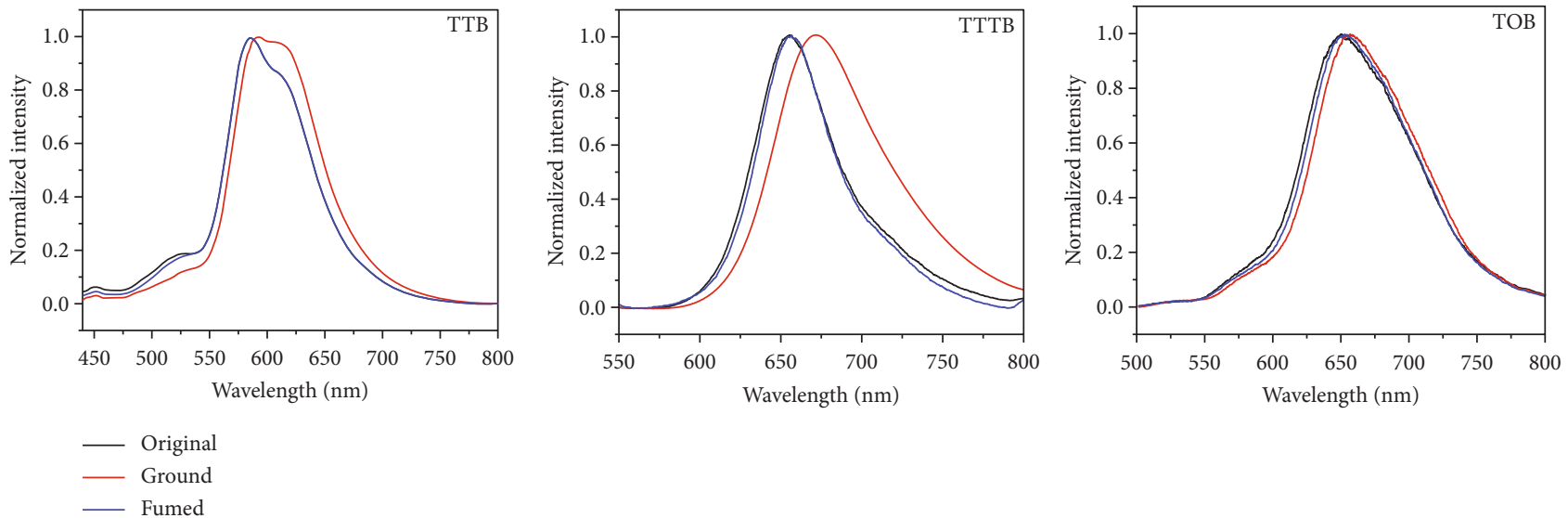

Figure 9: Normalized fluorescent spectra of TTB, TTTB, and TOB in different solid states: original nanoaggregate, ground, and fumed (excitation wavelength, TTB: $410 \mathrm{~nm}$, TTTB: $530 \mathrm{~nm}$, and TOB: $440 \mathrm{~nm}$ ).
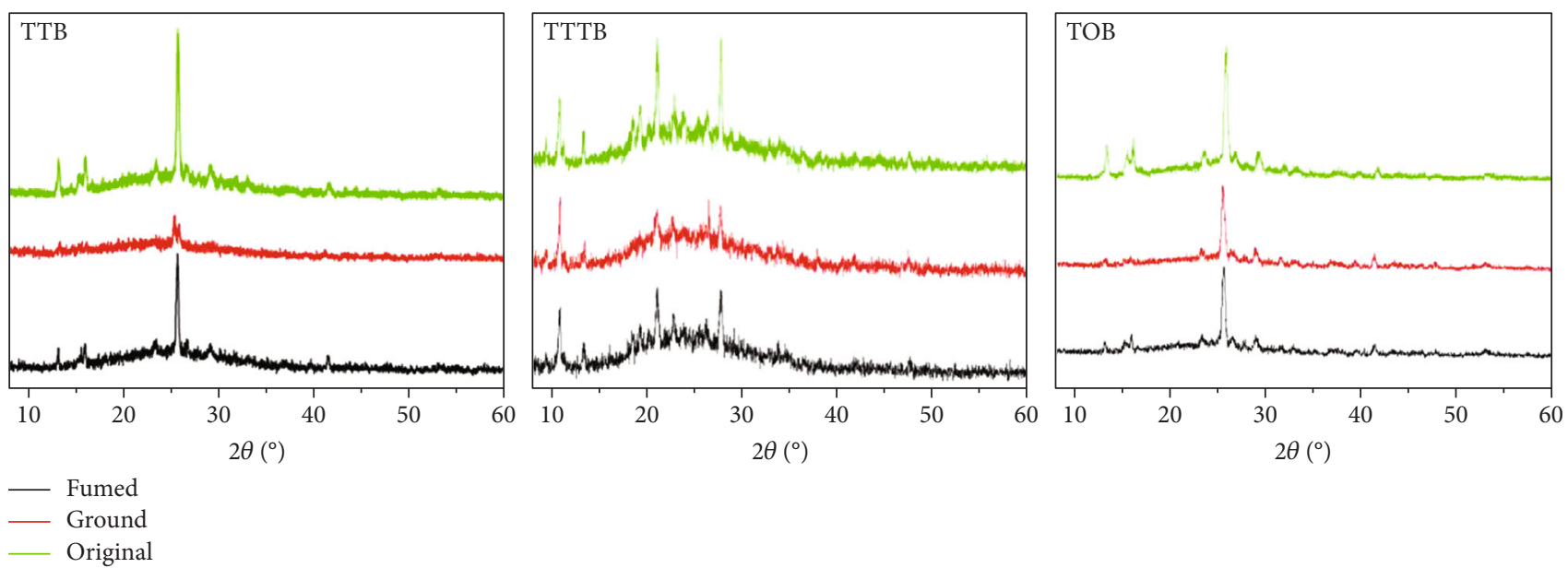

FIGURE 10: XRD patterns of TTB, TTTB, and TOB in different solid states: original, ground, and fumed.

the XRD patterns gained their initial characteristic peaks, indicating that the crystal structures were restored (Figure 10).

\section{Conclusions}

In summary, we reported three compounds (TTB, TTTB, and TOB) with typical AIE characteristics and highly reversible piezochromic and vapochromic properties. Among them, TTTB displayed the largest MFC spectra shift $(26 \mathrm{~nm})$ with visible color changes. It was confirmed that the emission bands of these compounds and their intensity dramatically changed by switching the nanoaggregates between crystalline and amorphous. These nanomaterials can be used in color emission and security [33].

\section{Data Availability}

The data used to support the findings of this study are available from the corresponding authors upon request.

\section{Conflicts of Interest}

The authors declare that they have no conflicts of interest.

\section{Acknowledgments}

This work was financially supported by the Natural Science Foundation of Shandong Province (ZR2018MB025), the Key R\&D Program Projects of Shandong Province (2017GGX202001), and the Funds for Research Leader in Jinan (2018GXRC028).

\section{Supplementary Materials}

Figure S1: the 1H-NMR spectra of TTB. Figure S2: the $1 \mathrm{H}-\mathrm{NMR}$ spectra of TTTB. Figure S3: the $1 \mathrm{H}-\mathrm{NMR}$ spectra of TOB. Figure S4: the 13C-NMR spectra of TTB. Figure S5: the 13C-NMR spectra of TTTB. Figure S6: the 13C-NMR spectra of TOB. Figure S7: the mass spectra of TTB. Figure S8: the mass spectra of TTTB. Figure S9: the mass spectra of TOB. (Supplementary Materials) 


\section{References}

[1] J. Luo, Z. Xie, J. W. Y. Lam et al., "Aggregation-induced emission of 1-methyl-1,2,3,4,5-pentaphenylsilole," Chemical Communications, vol. 18, no. 18, pp. 1740-1741, 2001.

[2] A. Qin, C. K. W. Jim, Y. Tang et al., "Aggregation-enhanced emissions of intramolecular excimers in disubstituted polyacetylenes," Journal of Physical Chemistry B, vol. 112, no. 31, pp. 9281-9288, 2008.

[3] Y. Hong, J. W. Y. Lam, and B. Z. Tang, "Aggregation-induced emission: phenomenon, mechanism and applications," Chemical Communications, vol. 29, no. 29, pp. 4332-4353, 2009.

[4] Y. Hong, J. W. Y. Lam, and B. Z. Tang, "Aggregation-induced emission," Chemical Society Reviews, vol. 40, no. 11, pp. 53615388, 2011.

[5] Y. Zhao, S. He, J. Yang et al., "Study on TICT emission of TPE-BODIPY derivatives mediated by methyl group on BODIPY," Optical Materials, vol. 81, pp. 102-108, 2018.

[6] J. Chai, Y. Wu, B. Yang, and B. Liu, "The photochromism, light harvesting and self-assembly activity of a multi-function Schiff-base compound based on the AIE effect," Journal of Materials Chemistry C, vol. 6, no. 15, pp. 4057-4064, 2018.

[7] G. Sych, J. Simokaitiene, O. Bezvikonnyi et al., "Exciplexenhanced singlet emission efficiency of nondoped organic light emitting diodes based on derivatives of tetrafluorophenylcarbazole and tri/tetraphenylethylene exhibiting aggregationinduced emission enhancement," The Journal of Physical Chemistry C, vol. 122, no. 26, pp. 14827-14837, 2018.

[8] I. Hladka, D. Volyniuk, O. Bezvikonnyi et al., "Polymorphism of derivatives of tert-butyl substituted acridan and perfluorobiphenyl as sky-blue OLED emitters exhibiting aggregation induced thermally activated delayed fluorescence," Journal of Materials Chemistry C, vol. 6, no. 48, pp. 13179-13189, 2018.

[9] G. Grybauskaite-Kaminskiene, D. Volyniuk, V. Mimaite et al., "Aggregation-Enhanced Emission and Thermally Activated Delayed Fluorescence of Derivatives of 9-Phenyl-9H-Carbazole: Effects of Methoxy and tert-Butyl Substituents," Chemistry - A European Journal, vol. 24, no. 38, pp. 9581-9591, 2018.

[10] M. Chen, L. Li, H. Wu et al., "Unveiling the different emission behavior of polytriazoles constructed from pyrazine-based AIE monomers by click polymerization," ACS Applied Materials \& Interfaces, vol. 10, no. 15, pp. 12181-12188, 2018.

[11] Z. Song, X. Lv, L. Gao, and L. Jiang, "Dramatic differences in the fluorescence of AIEgen-doped micro- and macrophase separated systems," Journal of Materials Chemistry C: Materials for Optical and Electronic Devices, vol. 6, no. 1, pp. 171-177, 2018.

[12] G. Liu, D. Q. Feng, D. Hua, T. Liu, G. Qi, and W. Wang, "Fluorescence Enhancement of Terminal Amine Assembled on Gold Nanoclusters and Its Application to Ratiometric Lysine Detection," Langmuir, vol. 33, no. 51, pp. 14643-14648, 2017.

[13] K. Li, X. Su, Y. Wang et al., "D- $\pi$-A type barbituric derivatives: aggregation induced emission, mechanofluorochromic and solvatochromic properties," Journal of Luminescence, vol. 203, pp. 50-58, 2018.

[14] Y. Sagara and T. Kato, "Mechanically induced luminescence changes in molecular assemblies," Nature Chemistry, vol. 1, no. 8, pp. 605-610, 2009.

[15] A. Li, Y. Liu, L. Han et al., "Pressure-induced remarkable luminescence-changing behaviours of 9, 10-distyrylanthracene and its derivatives with distinct substituents," Dyes and Pigments, vol. 161, pp. 182-187, 2019.

[16] Y. Zhang, J. Zhang, J. Shen et al., "Solid-State TICT-Emissive Cruciform: Aggregation-Enhanced Emission, Deep-Red to Near-Infrared Piezochromism and Imaging In Vivo," Advanced Optical Materials, vol. 6, no. 22, article 1800956, 2018.

[17] A. Dey, A. Garai, V. Gude, and K. Biradha, "Thermochromic, solvatochromic, and piezochromic $\mathrm{Cd}(\mathrm{II})$ and $\mathrm{Zn}(\mathrm{II})$ coordination polymers: detection of small molecules by luminescence switching from blue to green," Crystal Growth \& Design, vol. 18, no. 10, pp. 6070-6077, 2018.

[18] G. A. Crosby and J. N. Demas, "Measurement of photoluminescence quantum yields. Review," The Journal of Physical Chemistry, vol. 75, no. 8, pp. 991-1024, 1971.

[19] M. A. Ismail, "An efficient synthesis of 5 '-(4-cyanophenyl)$2,2^{\prime}$-bifuran-5-carbonitrile and analogues," Journal of Chemical Research, vol. 2006, no. 11, pp. 733-737, 2006.

[20] P. Y. Gu, C. J. Lu, Z. J. Hu et al., "The AIEE effect and twophoton absorption (TPA) enhancement induced by polymerization: synthesis of a monomer with ICT and AIE effects and its homopolymer by ATRP and a study of their photophysical properties," Journal of Materials Chemistry C: Materials for Optical and Electronic Devices, vol. 1, no. 14, pp. 2599-2606, 2013.

[21] K. Li, Y. Zhang, B. Qiao et al., "Facile fabrication of AIE/AIEEactive fluorescent nanoparticles based on barbituric for cell imaging applications," RSC Advances, vol. 7, no. 48, pp. 30229-30241, 2017.

[22] T. Han, X. Gu, J. W. Y. Lam et al., "Diaminomaleonitrile-based Schiff bases: aggregation-enhanced emission, red fluorescence, mechanochromism and bioimaging applications," Journal of Materials Chemistry C, vol. 4, no. 44, pp. 10430-10434, 2016.

[23] S. Mukherjee and P. Thilagar, "Molecular flexibility tuned emission in "V" shaped naphthalimides: $\mathrm{Hg}(\mathrm{II})$ detection and aggregation-induced emission enhancement (AIEE)," Chemical Communications, vol. 49, no. 66, pp. 7292-7294, 2013.

[24] K. Fujii, N. Iyi, R. Sasai, and S. Hayashi, "Preparation of a novel luminous heterogeneous system: rhodamine/coumarin/phyllosilicate hybrid and blue shift in fluorescence emission," Chemistry of Materials, vol. 20, no. 9, pp. 2994-3002, 2008.

[25] H. Shi, N. Zhao, D. Ding, J. Liang, B. Z. Tang, and B. Liu, "Fluorescent light-up probe with aggregation-induced emission characteristics for in vivo imaging of cell apoptosis," Organic \& Biomolecular Chemistry, vol. 11, no. 42, pp. 72897296, 2013.

[26] J. Demasa and G. Crosby, "Measurement of photoluminescence quantum yields," Chemistry International, vol. 37, no. 5-6, p. 31, 2015.

[27] W. Dong, J. Pina, Y. Pan, E. Preis, J. S. Seixas de Melo, and U. Scherf, "Polycarbazoles and polytriphenylamines showing aggregation-induced emission (AIE) and intramolecular charge transfer (ICT) behavior for the optical detection of nitroaromatic compounds," Polymer, vol. 76, pp. 173-181, 2015.

[28] Z. Chi, X. Zhang, B. Xu et al., "Recent advances in organic mechanofluorochromic materials," Chemical Society Reviews, vol. 41, no. 10, pp. 3878-3896, 2012.

[29] X. Q. Zhang, Z. G. Chi, B. J. Xu et al., "Comparison of responsive behaviors of two cinnamic acid derivatives containing 
carbazolyl triphenylethylene," Journal of Fluorescence, vol. 21, no. 1, article 697, pp. 133-140, 2011.

[30] X. F. Li, Z. G. Chi, B. J. Xu et al., "Synthesis and characterization of triphenylethylene derivatives with aggregationinduced emission characteristics," Journal of Fluorescence, vol. 21, no. 5, article 896, pp. 1969-1977, 2011.

[31] P. Xue, J. Ding, P. Wang, and R. Lu, "Recent progress in the mechanochromism of phosphorescent organic molecules and metal complexes," Journal of Materials Chemistry C, vol. 4, no. 28, pp. 6688-6706, 2016.

[32] Y. Jiang, G. Li, D. Zhu, Z. Su, and M. R. Bryce, “An AIE-active phosphorescent Ir (III) complex with piezochromic luminescence (PCL) and its application for monitoring volatile organic compounds (VOCs)," Journal of Materials Chemistry C, vol. 5, no. 46, pp. 12189-12193, 2017.

[33] S. Lu, G. Xiao, L. Sui et al., "Piezochromic carbon dots with two-photon fluorescence," Angewandte Chemie International Edition, vol. 56, no. 22, pp. 6187-6191, 2017. 


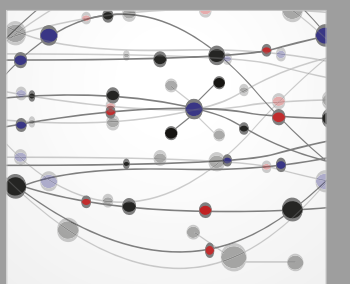

The Scientific World Journal
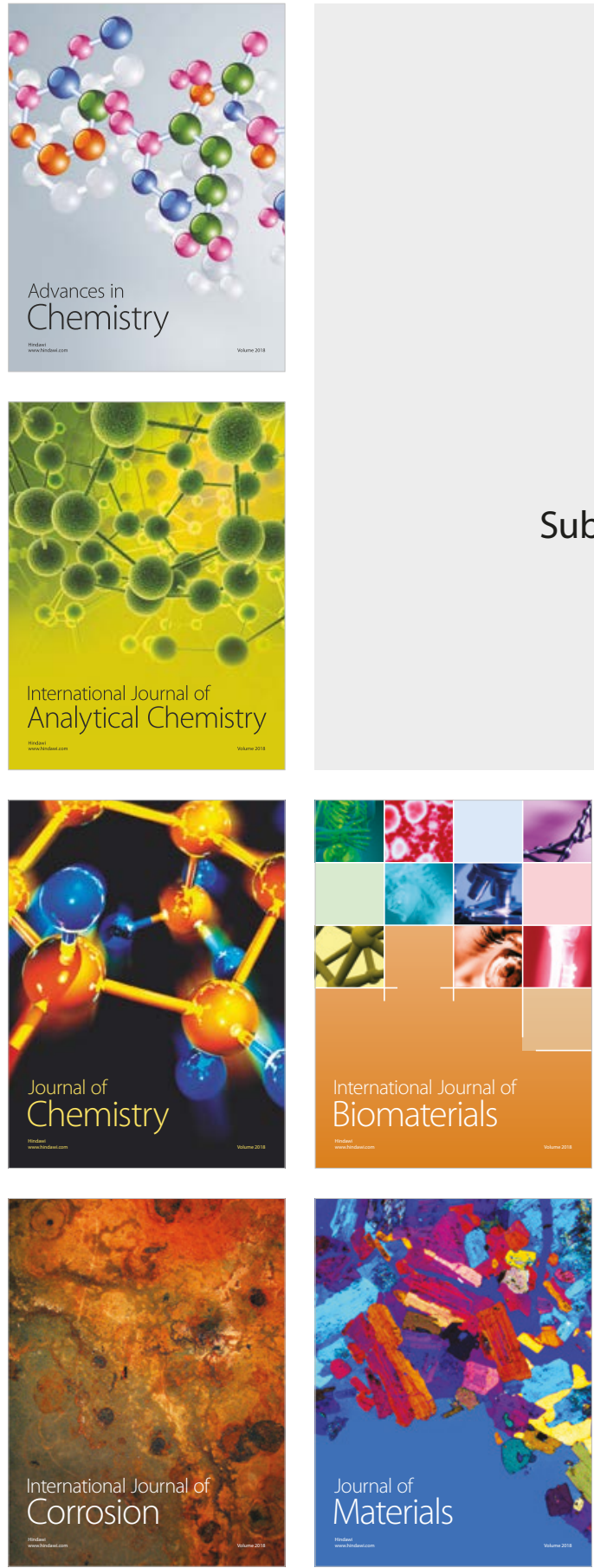

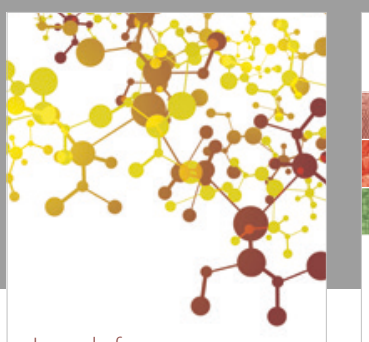

Journal of

Applied Chemistry
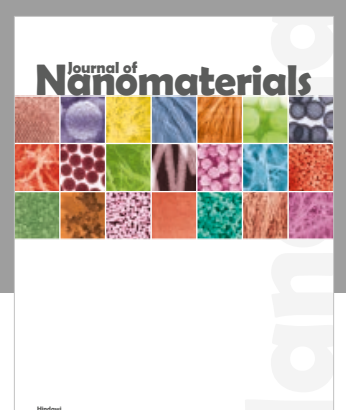

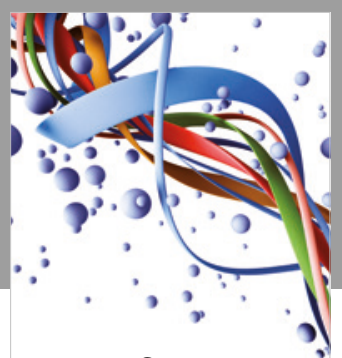

Scientifica

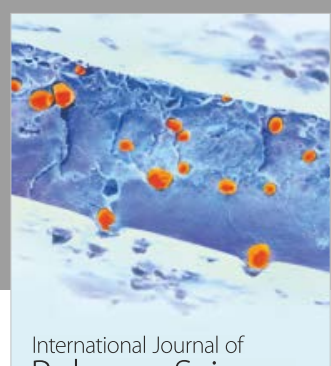

Polymer Science

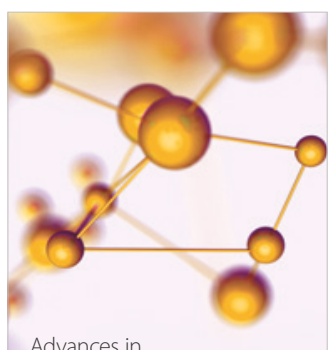

Physical Chemistry
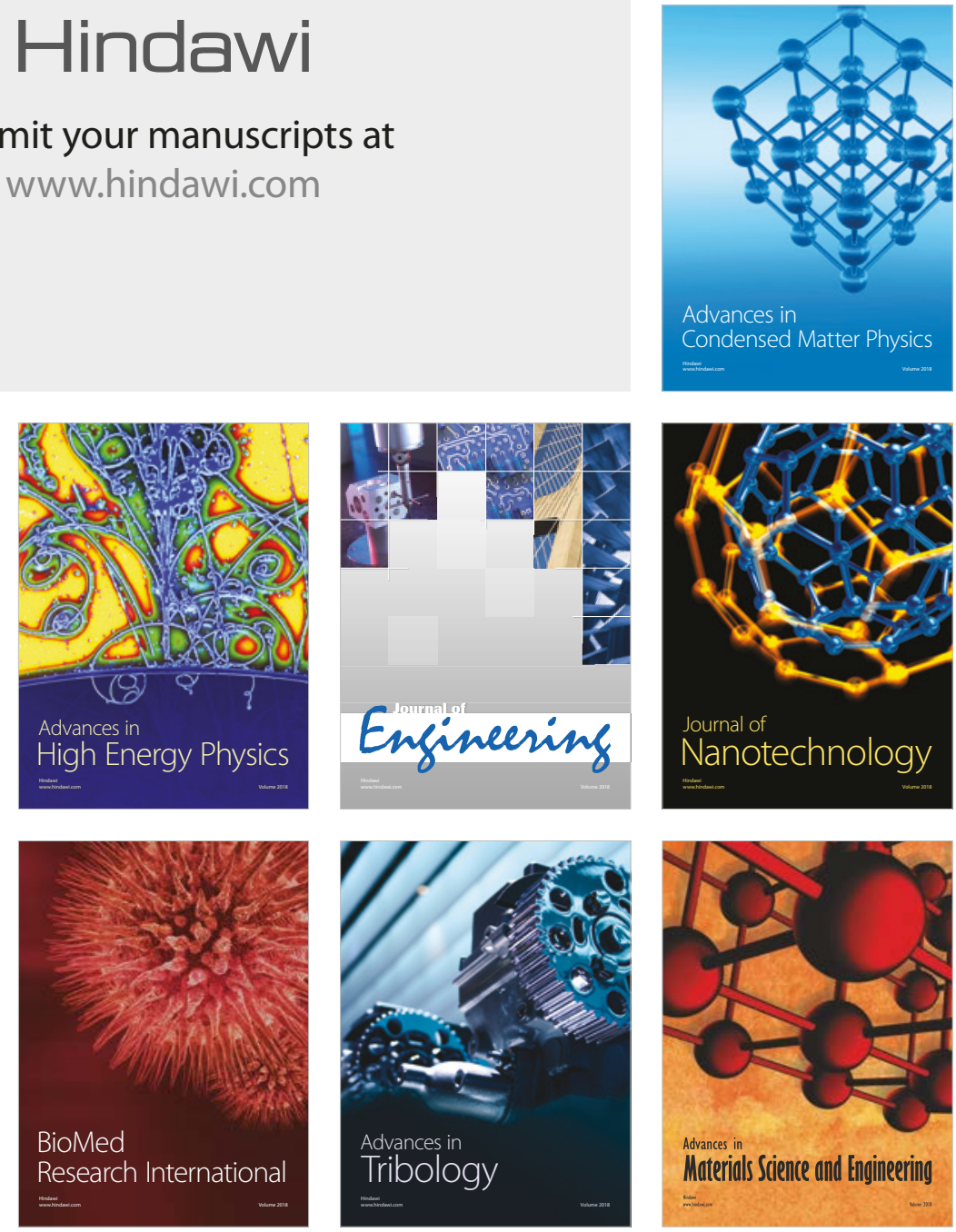